Bull. Mater. Sci., Vol. 21, No. 4, August 1998, pp. 297-301. (C Indian Academy of Sciences.

\title{
CVT growth of zirconium sulphoselenide single crystals
}

\author{
S G PATEL*, S K ARORA and M K AGARWAL \\ Department of Physics, Sardar Patel University, Vallabh Vidyanagar 388 120, India \\ MS received 22 January 1996; revised 4 February 1998
}

\begin{abstract}
Large single crystals of a few important members $(x=0,1,2)$ of the series $\mathrm{ZrS}_{x} \mathrm{Se}_{2-x}$ compounds have been obtained by chemical vapour (iodine) transport method. The crystals have been characterized for several properties. Their semiconducting nature is inferred from resistivity vs temperature measurements, their optical absorption data reveal indirect gap transitions, their thermoscans indicate their stability over a limited temperature range, and they essentially grow by the mechanism of two dimensional layer propagation.
\end{abstract}

Keywords. CVT growth; zirconium sulphoselenide.

\section{Introduction}

The need for developing a cheap yet effective method of converting solar energy into electrical or chemical energy stimulated rapid advancement of semiconductor electrochemistry in the past decades and as a result certain useful materials, for example, metallic chalcogenides which have potential in photovoltaic and photoelectrochemical solar cell studies have attracted attention of crystal growers. Among them, a typical class of compounds, viz. $\mathrm{ZrS}_{x} \mathrm{Se}_{2-x}$ have been shown to be important as high temperature high pressure lubricants, catalysts and switching devices. Single crystals of these materials have been grown, e.g. $\mathrm{ZrSe}_{2}$ (Nitsche et al 1961; Greenaway and Nitsche 1965; Rimington et al 1972; Whitehouse et al 1973; Zheng et al 1989; Akaike et al 1994), $\mathrm{ZrS}_{2}$ (Greenaway and Nitsche 1965; Conroy and Park 1968; Rimington et al 1972; Terashima and Imai 1987) using halogen transport. We report here the single crystal growth and a few essential data of the members of the series $\mathrm{ZrS}_{x} \mathrm{Se}_{2-x}(x=0,1,2)$.

\section{Experimental}

With a view to allowing effective and faster transport of constituents to produce the necessary supersaturation for crystal growth in a vapour phase system, the chemical (iodine) vapour transport method was employed. A twozone muffle furnace was fabricated for this purpose, having typical temperature profile as shown in figure 1 . For preparing the starting material from available powders of zirconium (99.7\% purity, Koch-Light Labs), sulphur (99.99\% purity, Koch-Light Labs) and selenium (99.99\% purity, Johnson Matthey), corresponding to a stoichio-

*Author for correspondence metric charge of $10 \mathrm{~g}$ were introduced into a thoroughly cleaned quartz ampoule and sealed at a pressure of $10^{-5}$ torr. Care was, however, taken in vigorously shaking the ampoule so as to distribute the mixture along the entire length. This loaded ampoule was coaxially placed into the tube furnace (figure 2), whose temperature was slowly increased to $1073 \mathrm{~K}$ and maintained for $72 \mathrm{~h}$. Then the furnace was shut down to bring it to room temperature. The resulting brilliant, dark powder was transferred to another evacuated quartz ampoule with $5 \mathrm{mg} \mathrm{cm}^{-3}$ iodine. This charged ampoule was placed again coaxially in the preset furnace. The desired zone temperatures (figure 1) were gradually attained and after the desired optimized growth period, the furnace was cooled down slowly at $50 \mathrm{~K} / \mathrm{h}$ to room temperature. The details of growth conditions and the results are given in table 1 .

The crystals obtained had plate-like morphology (ab-plane), with c-axis normal to the plate. The growth conditions have been found to be so critical that, as was observed, reducing the zone temperature by $10 \mathrm{~K}$ from the optimized values resulted in the formation of red monoclinic needles of $\mathrm{ZrS}_{3}$ (Patel et al 1993). This observation is in agreement with that of Whitehouse et al (1973) and Al-Alamy et al (1977). The surfaces of $\mathrm{ZrS}_{x} \mathrm{Se}_{2-x}$ crystals as obtained, were cleared of iodine contamination by heating in SICO oven kept overnight at $400 \mathrm{~K}$.

\section{Characterization}

Some of the crystals obtained in the growth runs are shown in figure 3 . Unit cell parameters of the grown crystals have been measured from $\mathrm{X}$-ray powder diffractograms. The values of ' $a$ ' and ' $c$ ' parameters obtained from XRD traces, the axial ratio c/a and X-ray density 
for all the samples have been shown in table 2 . It is observed that the value of lattice parameter ' $a$ ' varies linearly with composition obeying the relation (1),

$$
\mathrm{a}(x)=3.758-0.049 x,
$$

but the $c$ parameter varies non-linearly obeying the relation (2), so does the $\mathrm{X}$-ray density.

$$
c(x)=6.116-0.87 x-0.036 x^{2} .
$$

This non-linearity is in confirmation with the observations made by Whitehouse et al (1973) in $\mathrm{ZrS}_{x} \mathrm{Se}_{2-x}$.

The results of EDAX analysis confirm the nonstoichiometry of the grown crystals. The nonstoichiometry in these crystals is a most commonly observed feature (Whitehouse and Balchin 1978; Ikari et al 1995), which can be attributed to the inelastic lattice distortion produced by transfer of metal cations into the vacant sites which are created by chalcogen atoms into the van der Waals gap, as has also been argued upon by Gleizes and Jeannin (1970).

For electrical characterization, the resistivity measurements perpendicular to c-axis, i.e. along the basal plane, $\rho_{\text {III }}$, using the four-probe technique were made by making four pressure contacts on the basal $(a, b)$ plane. The samples after making contacts were placed in a small

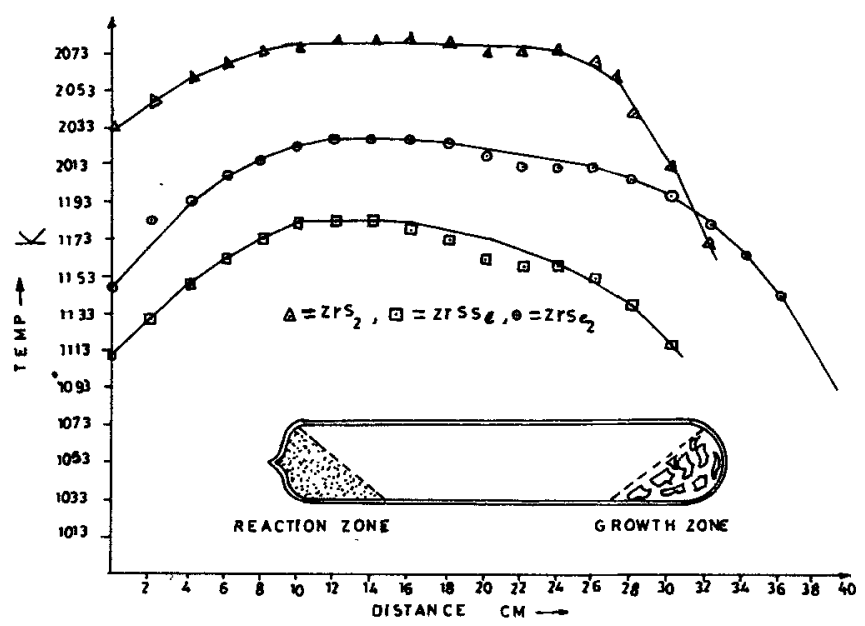

Figure 1. The temperature profile for the growth of $\mathrm{ZrS}_{x} \mathrm{Se}_{2-x}(x=0,1$ and 2$)$. oven with a temperature range $300-450 \mathrm{~K}$. The values of room temperature resistivity and the activation energies obtained from the plots of $\log \rho_{\|}$vs $1 / T$ for $\mathrm{ZrS}_{x} \mathrm{Se}_{2-x}$ $(x=0,1,2)$ are shown in table 2 . It is seen that the resistivity shows an increase with increase in the sulphur content.

The resistivity perpendicular to basal plane $\rho_{\perp}$ i.e. parallel to $\mathrm{c}$-axis, was investigated by taking two pressure contacts, one above and the other below the specimen, in a direction perpendicular to the basal plane. The values of $\rho_{\|}$and $\rho_{\perp}$ at room temperature were used to determine the anisotropy ratio for all the three compounds and the values thus attained are also presented in table 2. It is observed that $\mathrm{ZrS}_{x} \mathrm{Se}_{2-x}$ becomes more anisotropic with increasing amount of sulphur. In comparison to $\mathrm{ZrSe}_{2}$, there is no significant change in anisotropy in $\mathrm{ZrSSe}$. But upon suppressing selenium altogether in $\mathrm{ZrS}_{x} \mathrm{Se}_{2-x}$, the anisotropy ratio shows a dramatic increase. This clearly indicates that increasing the amount of sulphur makes the compounds more anisotropic.

The thermoelectric power (TEP) measurements were carried out with the thermopower set up TPSS-200 developed by Scientific Solutions, Mumbai. In all the measurements, $\Delta T$ was kept as $4 \mathrm{~K}$. The values of TEP for all the three crystals are given in table 2 . The sign of TEP for all the samples was found to be negative, indicating their $n$-type nature. We can clearly notice the increase in the value of TEP with an increase in the

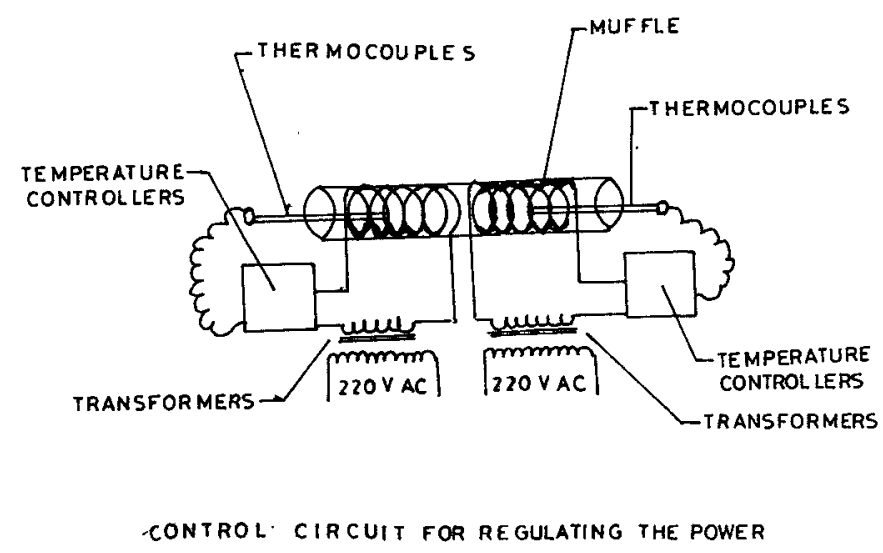

Figure 2. A schematic diagram showing the control circuit for regulating the power to the two-zone crystal growth furnace.

Table 1. Growth parameters for single crystals of $\mathrm{ZrS}_{x} \mathrm{Se}_{2-x}$.

\begin{tabular}{cccccl}
\hline $\begin{array}{c}\text { Composition } \\
X\end{array}$ & $\begin{array}{c}\text { Reaction } \\
\text { temperature } \\
(\mathrm{K})\end{array}$ & $\begin{array}{c}\text { Growth } \\
\text { temperature } \\
(\mathrm{K})\end{array}$ & $\begin{array}{c}\text { Growth } \\
\text { time } \\
(\mathrm{h})\end{array}$ & $\begin{array}{c}\text { Crystal size } \\
(\mathrm{mm} \times \mathrm{mm} \times \mathrm{mm})\end{array}$ & Appearance \\
\hline 0 & 1123 & 1073 & 500 & $12 \times 10 \times 0.20$ & Dark bronze green \\
1 & 1153 & 1123 & 140 & $13 \times 11 \times 0.09$ & Violet metallic \\
2 & 1283 & 1203 & 450 & $10 \times 09 \times 0.32$ & Violet brown \\
\hline
\end{tabular}


value of $x$ in $\mathrm{ZrS}_{x} \mathrm{Se}_{2-x}$. This increase in the value of TEP with $x$ makes the crystals more resistive. That this is true can be seen from the room temperature resistivities for these compounds in table 2 .

The optical absorption data were analysed in terms of the theory of Bardeen et al (1956),

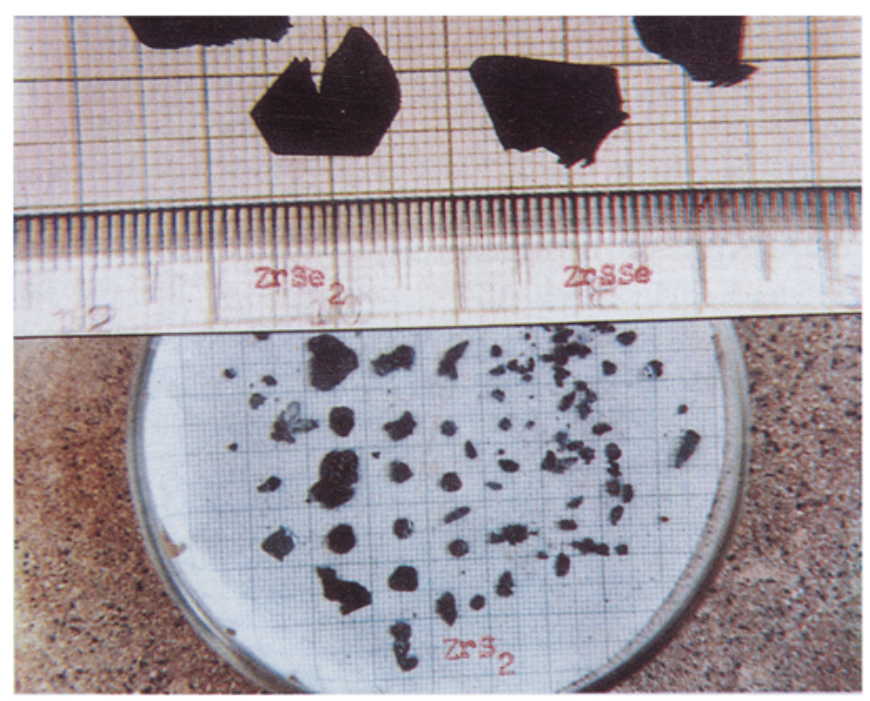

Figure 3. As grown single crystals of $\mathrm{ZrSe}_{2}, \mathrm{ZrSSe}$ and $\mathrm{ZrS}_{2}$ separated in a petri dish with a centimeter graph paper.

$$
\alpha=\beta\left(h v-E_{\mathrm{g}}\right)^{\mathrm{r}} / h \nu,
$$

where the symbols have their usual meaning. Thus band gaps were obtained from the graphical plots of $(\alpha h \nu)^{1 / 2}$ vs $h v$, employing a dual beam UV-VIS 'Schimadzu' spectrophotometer in the wavelength range 190-2500 nm at room temperature. $E_{\mathrm{g}}$ (see table 2) was found to increase linearly with the composition $x$, and there exist indirect transitions in the crystals. It is implied that the three members, $x=0,1$ and 2 of the family $\mathrm{ZrS}_{x} \mathrm{Se}_{2-x}$ possess similarity in the bond configuration with $\mathrm{Zr}$.

Thermograms of $\mathrm{ZrS}_{x} \mathrm{Se}_{2-x}(x=0,1,2)$ were scanned at a heating rate of $10 \mathrm{~K} \mathrm{~min}^{-1}$. The values of activation energies obtained from the analysis of the thermograms (table 2) can be placed in the following order

$$
\mathrm{ZrS}_{2}>\mathrm{ZrSSe}>\mathrm{ZrSe}_{2} .
$$

This suggests that the stability of the compound increases with the increase in the sulphur content. The thermal decomposition was found to be in the first order and to follow Arrhenius type of kinetics having activation energy in the range $10-50 \mathrm{~kJ} \mathrm{~mol}^{-1} . \mathrm{ZrS}_{2}$ decomposes at $800 \mathrm{~K}$ to form $\mathrm{Zr}_{3} \mathrm{~S}_{4}$, followed by the formation of $\mathrm{ZrO}_{2}$ at $900 \mathrm{~K}$, whence $\mathrm{ZrO}_{2}$ and $\mathrm{ZrS}_{4}$ coexist as a two-phase mixture. At $950 \mathrm{~K}$, some of the tetragonal $\mathrm{ZrO}_{2}$ is converted into monoclinic form, resulting into 3-phase mixture: $\mathrm{Zr}_{3} \mathrm{~S}_{4}+\mathrm{ZrO}_{2}$ (tetragonal) $+\mathrm{ZrO}_{2}$ (monoclinic), which remains stable up to around $1200 \mathrm{~K}$.

\begin{tabular}{|c|c|c|c|}
\hline Property & $\mathrm{ZrSe}_{2}$ & $\mathrm{ZrSSe}$ & $\mathrm{ZrS}_{2}$ \\
\hline ' $\mathrm{a}$ ' (A) & 3.758 & 3.709 & 3.660 \\
\hline 'c' (A) & $6 \cdot 116$ & 5.996 & $5 \cdot 805$ \\
\hline c/a ratio & 1.627 & 1.619 & 1.586 \\
\hline $\begin{array}{l}\mathrm{X} \text {-ray density } \\
\left(\mathrm{g} \mathrm{cm}^{-3}\right)\end{array}$ & 5.53 & 4.71 & 3.83 \\
\hline $\begin{array}{l}\text { Room temperature resistivity } \\
\text { along the basal plane }\left(\rho_{\|}\right) \\
\left(\times 10^{-4} \mathrm{~cm} \Omega\right)\end{array}$ & $23 \cdot 40$ & $47 \cdot 3$ & 796.7 \\
\hline $\begin{array}{l}\text { Room temperature resistivity } \\
\text { perpendicular to basal plane }\left(\rho_{\perp}\right) \\
(\mathrm{cm} \Omega)\end{array}$ & 8.02 & 34.62 & $4.655 \times 10^{4}$ \\
\hline Anisotropy $\left(\rho_{\perp} \rho_{\|}\right)$ & $3.43 \times 10^{3}$ & $7.319 \times 10^{3}$ & $5.843 \times 10^{5}$ \\
\hline $\begin{array}{l}\text { Electrical activation energy } \\
(\mathrm{eV})\end{array}$ & 0.059 & 0.023 & 0.018 \\
\hline $\begin{array}{l}\text { Thermoelectric power (TEP) } \\
\left(\mathrm{mV} \mathrm{K}^{-1}\right)\end{array}$ & 0.275 & 0.32 & 0.49 \\
\hline $\begin{array}{l}\text { Optical band gap, } E_{g} \\
(\mathrm{eV})\end{array}$ & 1.20 & 1.44 & 1.72 \\
\hline $\begin{array}{l}\text { Thermal activation energy } \\
(\mathrm{kJ} / \mathrm{mol})\end{array}$ & $12 \cdot 26$ & 22.76 & 30.09 \\
\hline
\end{tabular}

Table 2. Some characteristic data on $\mathrm{ZrS}_{x} \mathrm{Se}_{2-r}$ crystals. 


\section{Growth mechanism}

The morphology of a crystal surface, according to Newmann principle, under near equilibrium condition reflects the inherent crystal symmetry and consequently

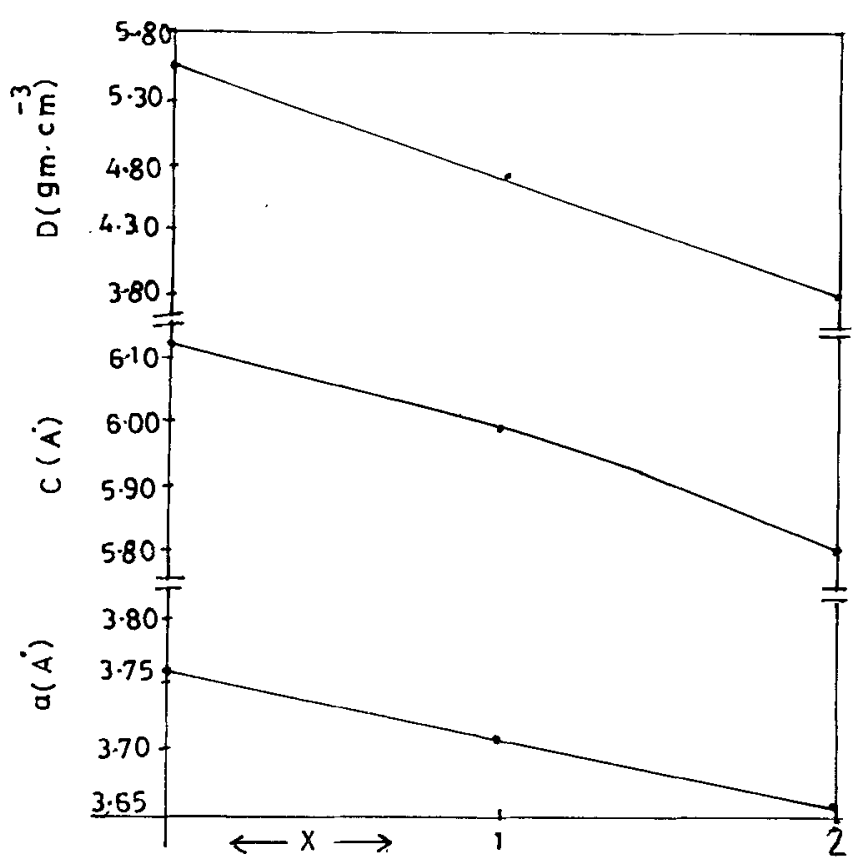

Figure 4. Graphs showing variations of lattice parameters a, $c$ and X-ray density $D$ with composition $x$ for the series $\mathrm{ZrS}_{x} \mathrm{Se}_{2-x}$ (with $x=0,1$ and 2 ). the as-grown crystal surfaces often exhibit some features which signify how the crystals grow. It was thought worthwhile to examine crystal surfaces to derive appropriate mechanism responsible for crystal growth to take place.

The faces on the grown single crystals of $\mathrm{ZrS}_{2}, \mathrm{ZrSe}_{2}$ and ZrSSe were examined under 'Epignost' optical microscope, and since no crystal was found to contain any kind of spiral traces, we strongly believe that $\mathrm{ZrS}_{x} \mathrm{Se}_{2-x}$ crystals do not grow by screw dislocation mechanism. Typical surface features of the kind shown in figure $5(\times 450)$ were frequently observed on the as grown basal faces of $\mathrm{ZrSe}_{2}$ crystals. One can identify the surface to be flat and smooth, accompanied by some growth layers initiating from crystal edges and boundary. The left half of the crystal face in figure 5 shows more bunching and crowding of layers than in the middle. This is attributed to exhaustion of nutrients and hence fall of supersaturation at the end stage of growth in the ampoule. Microstructures of this kind were seen in $\mathrm{ZrSSe}$ and $\mathrm{ZrS}_{2}$ crystals also,

A flat face being the surest test of two dimensional layer theory, we are inclined to believe that zirconium sulphoselenides grow by the mechanism of piling and spreading of layers.

\section{Discussion}

Sulphur being non-metal, is less conducting than the other two elements viz. $\mathrm{Zr}$ and $\mathrm{Se}$, when incorporated with increasing amount into the lattice is likely to render

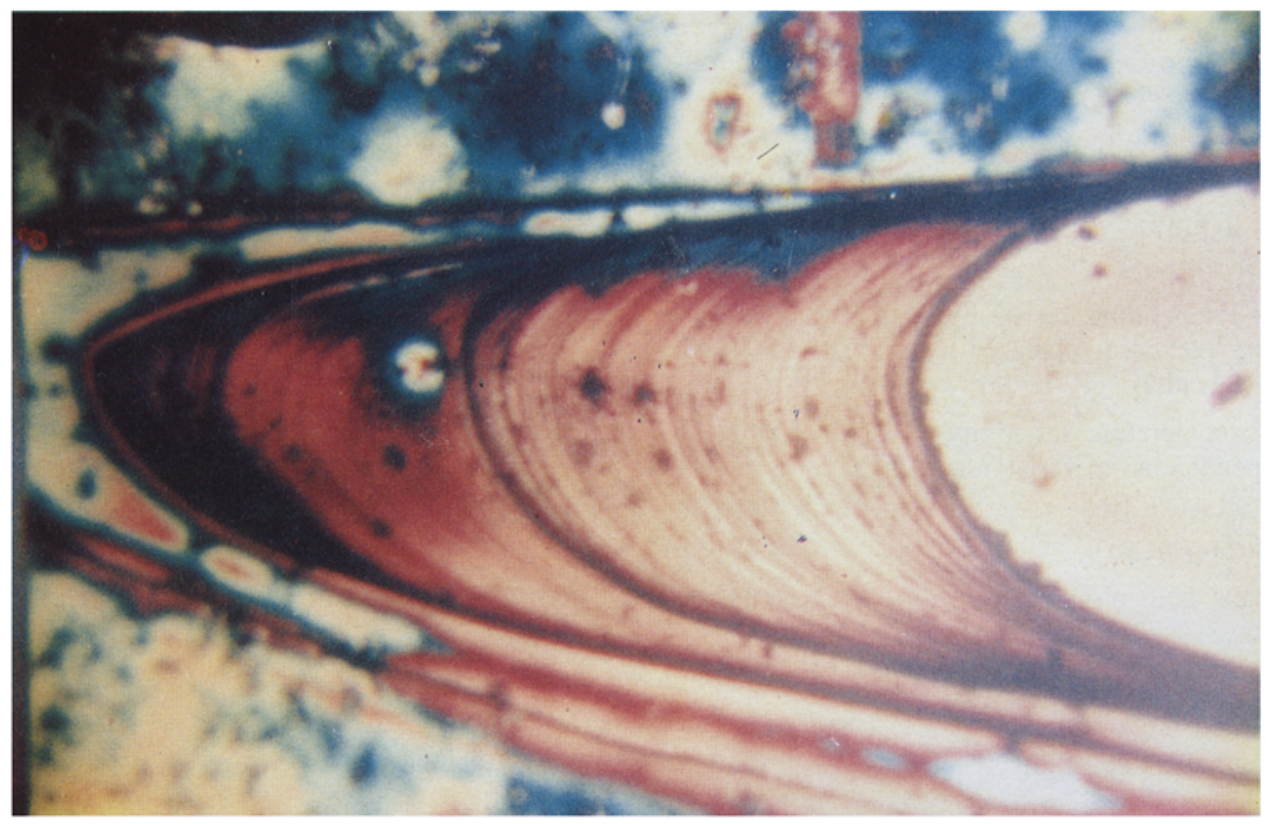

Figure 5. Micrograph showing flat surface as well as the presence of growth layers on as grown face of a $\mathrm{ZrSe}_{2}$ single crystal. 
the compound more resistive. This is practically what has been observed in the present case. Observations on electrical resistivity support this view. The increase in resistivity with increase in sulphur content, i.e. $x$ in $\mathrm{ZrS}_{x} \mathrm{Se}_{2-x}$ can be linked with increasing band gap with $x$.

The activation energies shown in table 2 are very low. This shows that the conduction in this temperature range is due to transitions from impurity levels lying within the band gap to the conduction band. There is no systematic variation of activation energy with sulphur content in $\mathrm{ZrS}_{x} \mathrm{Se}_{2-x}$. This shows that activation energy does not depend exclusively upon sulphur but is also due to the presence of impurities (for example, iodine) in the samples. In order that the variation of activation energy may depend upon sulphur content in the sample, the temperature range in which the resistivity variation should be considered must be very high so that activation energy obtained from the plots of $\log \rho_{\|}$vs $1 / T$ may be comparable with the band gap.

\section{Conclusions}

Coaxially loading the quartz ampoule in the furnace with critical end-temperatures maintained across the two zones helps to obtain larger size single crystals of $\mathrm{ZrS}_{x} \mathrm{Se}_{2-x}$. The electrical resistivity variation with temperature of $\mathrm{ZrS}_{x} \mathrm{Se}_{2-x}$ single crystals confirms their semiconductivity behaviour. The dependence of resistivity on sulphur content in the single crystals has been thoroughly explained. The resistivity measured in a direction parallel to c-axis clearly shows that $\mathrm{ZrS}_{x} \mathrm{Se}_{2-x}$ single crystals become more and more two dimensional with increase in the sulphur content. The resistivity anisotropy measurements on the $\mathrm{ZrS}_{x} \mathrm{Se}_{2-x}$ also support this conjecture. The thermoelectric power measurements show that the single crystals of $\mathrm{ZrS}_{4} \mathrm{Se}_{2-x}$ possess $n$-type semiconducting nature. A study of thermograms obtained from these crystals show that their stability increases with increase of sulphur atoms. The crystal growth has been found to occur by two dimensional layer propagation theory and the dislocation mechanism is inapplicable.

\section{Acknowledgement}

Authors are grateful to the University Grants Commission, New Delhi, for financial assistance.

\section{References}

Akaike Y, Iwaki S, Futagami K, Ikari T, Maeda K and Yokoyama H 1994 Bull. Faculty Engg. Miyazaki Univ. 4071

Al-Alamy F A S, Balchin A A and White M 1977 J. Mater. Sci. 122037

Bardeen J, Blatt F J and Hall L H 1956 in Photoconductivity conference, Atlantic City, N.J. (New York: Wiley) p. 146

Conroy L E and Park K C 1968 Inorg. Chem. 7459

Gleizes A and Jeannin $1970 \mathrm{~J}$. Solid State Chem. 1180

Greenaway D and Nitsche R 1965 J. Phys. Chem. Solids 26 1445

Ikari T, Maeda K, Futagami K and Nakashima A $1995 \mathrm{Jpn} J$. Appl. Phys. 341442

Nitsche R, Bolsterli H and Lichtensteiger M 1961 J. Phys. Chem. Solids 21199

Patel S G, Chaki S H and Agarwal Ajay 1993 Phys. Status Solidi (a) 140207

Rimmington H P B, Balchin A A and Tanner B K $1972 J$. Cryst. Growth 1551

Terashima K and Imai I 1987 Solid State Commun. 63315

Whitehouse C R and Balchin A A 1978 Phys. Status Solidi (a) $47 \mathrm{~K} 173$

Whitehouse C R, Rimmington H P B and Balchin A A 1973 Phys. Status Solidi (a) 18623

Zheng X U, Kuriyaki H and Hirakawa K 1989 J. Phys. Soc. Jpn 58622 